\title{
The Incidence and Cost of New Onset Hyperlipidemia Claims Among US Wait-Listed and Transplanted Renal Allograft Recipients
}

\author{
Robert S. Woodward ${ }^{*}, 1$, Timothy F. Page ${ }^{2}$, Andrea Menclova ${ }^{3}$, Mark A. Schnitzler ${ }^{4}$ and \\ Daniel C. Brennan ${ }^{5}$ \\ ${ }^{I}$ Department of Health Management and Policy, University of New Hampshire, 4 Library Way, Durham, NH 03824, \\ USA \\ ${ }^{2}$ Department of Health Policy and Management, Florida International University, 11200 SW 8 Street, Miami, FL 33199, \\ USA \\ ${ }^{3}$ Department of Economics and Finance, University of Canterbury, Private Bag 4800, Christchurch, New Zealand \\ ${ }^{4}$ School of Medicine, St. Louis University Medical Center, 1402 S. Grand Blvd., St. Louis, MO 63104, USA \\ ${ }^{5}$ Washington University, School of Medicine, 660 S. Euclid Ave., St. Louis, Mo. 63110, USA
}

\begin{abstract}
Background: Hyperlipidemia increases mortality and is common with kidney-disease. New-onset hyperlipidemia (NOHL) among patients wait-listed and after transplantation may impact costs and graft-survival of patients with kidney disease. Methods: Using the United States Renal Data System, we compared the costs to Medicare associated with or without NOHL in wait-listed patients in the second and first year pre-transplant and transplanted patients in the first and second year post-transplant. We also examined the impact on graft-survival of NOHL. Results: New onset hyperlipidemia was especially expensive when it occurred well before transplantation. When compared with individuals with no hyperlipidemia, patients with early onset hyperlipidemia cost an extra $\$ 15,228$ in the two years before transplantation and an extra $\$ 14,673$ in the two years following transplantation. As has been found in prior studies, patients without any NOHL had the worst graft survival rates. Conclusions: Although NOHL was associated with increased pre- and post-transplant costs, patients diagnosed with NOHL between the second year before and second year after transplantation experienced higher graft-survival rates than those without NOHL by 2-years post-transplantation. Prior studies attribute this relationship to inflammation and malnutrition, which result in lower cholesterol levels and worse outcomes.
\end{abstract}

Keywords: Renal transplantation, hyperlipidemia, graft survival.

\section{INTRODUCTION}

Hyperlipidemia, elevated blood lipid levels, is a prevalent condition among patients with end stage renal disease (ESRD) [1] and has been shown to be associated with maintenance immunosuppression [2], atherosclerosis and cardiovascular mortality [3-5]. New-onset hyperlipidemia (NOHL) may impact costs and graft-survival. This study builds on prior research that has evaluated the costs and outcomes associated with other diseases, such as diabetes, sepsis, and pneumonia [6-7].

An appropriate determination of the true incidence of post-transplant hyperlipidemia (PTHL) has been difficult. Satterthwaite reported one year incidences of hypercholesterolemia of $68 \%$ and $26 \%$ for renal transplant patients on cyclosporine- and tacrolimus-based immunosuppression, respectively [8]. In the US Multicenter Tacrolimus Renal Trial, the need for lipid lowering agents at three years was $39 \%$ and $14 \%$ for patients on cyclosporine- and tacrolimus-

*Address correspondence to this author at the Department of Health Management and Policy, University of New Hampshire, 4 Library Way, Durham, NH 03824, USA; Tel: (603) 862-7032; Fax: (603) 862-3461; E-mail: rsw@unh.edu based immunosuppression, respectively [9]. In the European Multicenter Tacrolimus Renal Trial, hypercholesterolemia at four years was present in $30 \%$ and $10 \%$ of patients on cyclosporine- and tacrolimus-based immunosuppression, respectively [10]. However, these studies used different definitions of hyperlipidemia, had varying patient populations, and employed varying immunosuppressive regimens. The current study identified the incidence of PTHL by comparing the incidence of hyperlipidemia among transplant recipients to that of patients on dialysis.

The appropriate measure of the incidence of PTHL actually attributable to transplantation should be based on the difference between the observed incidence of PTHL and the incidence which would have been expected to occur in a baseline similar population that had not been transplanted. "Wait-listed" patients, that subset of patients on dialysis eligible for a transplant but who have not yet received one, define the most appropriate comparison group when determining the baseline incidence of new onset hyperlipidemia (NOHL). While the incidence of NOHL in "wait-listed" patients has not been determined previously, the high correlation between hyperlipidemia and ESRD suggests that the incidence of NOHL among "wait-listed" patients may be higher than the incidence of NOHL in the general population 
[11]. At the same time, however, the relatively high health status of the "wait-listed" kidney patients suggests their NOHL incidence may be lower than that reported among the overall ESRD population.

The development of NOHL in wait-listed and PTHL in transplanted patients is likely to be associated with significant costs, due to the association among hyperlipidemia, cardiovascular and peripheral vascular complications [3-5]. Because Medicare is the primary payer for most patients with ESRD, the implications of the incidence and the costs of NOHL and PTHL and impact on graft-survival has national significance.

The primary objective of this study was to determine the costs of NOHL based on the time of onset relative to the time of transplantation. We used national data to calculate the incidence and Medicare payments associated with NOHL from two years pre-transplant to two years post-transplant. Each of these calculations was performed for patients with NOHL two years pre-transplant, one year pre-transplant, one year post-transplant, two years post-transplant, and for patients with no hyperlipidemia between two years pretransplant and two years post-transplant. In addition, we examined graft survival rates by the time of hyperlipidemia onset relative to the time of transplantation.

\section{METHODS}

We examined data from the 2003 release of the United States Renal Data System (USRDS) allowing for two-years of follow-up [12]. These data permit examination of the association of clinical outcomes with characteristics of donors, recipients, and protocols, and correlation with outcomes with the actual costs to Medicare.

In the 2003 release of the USRDS database, we identified 37,906 first, single-organ renal transplants from cadaveric donors to recipients 20-65 years old which were performed since 1995 and for which Medicare was the primary payer. Of the 37,906 subjects at transplant, 22,556 (59.5\%) were followed at 2 years (730 days) before transplant; 30,356 $(80.1 \%)$ were followed at 1 year (365 days) before transplant; 31,361 (82.7\%) were followed at 1 year after transplant; and 25,939 (68.4\%) were followed at two years after transplant (Fig. 1).

This research used the time of onset of NOHL, to construct patient cohorts. A diagnosis of NOHL was defined as
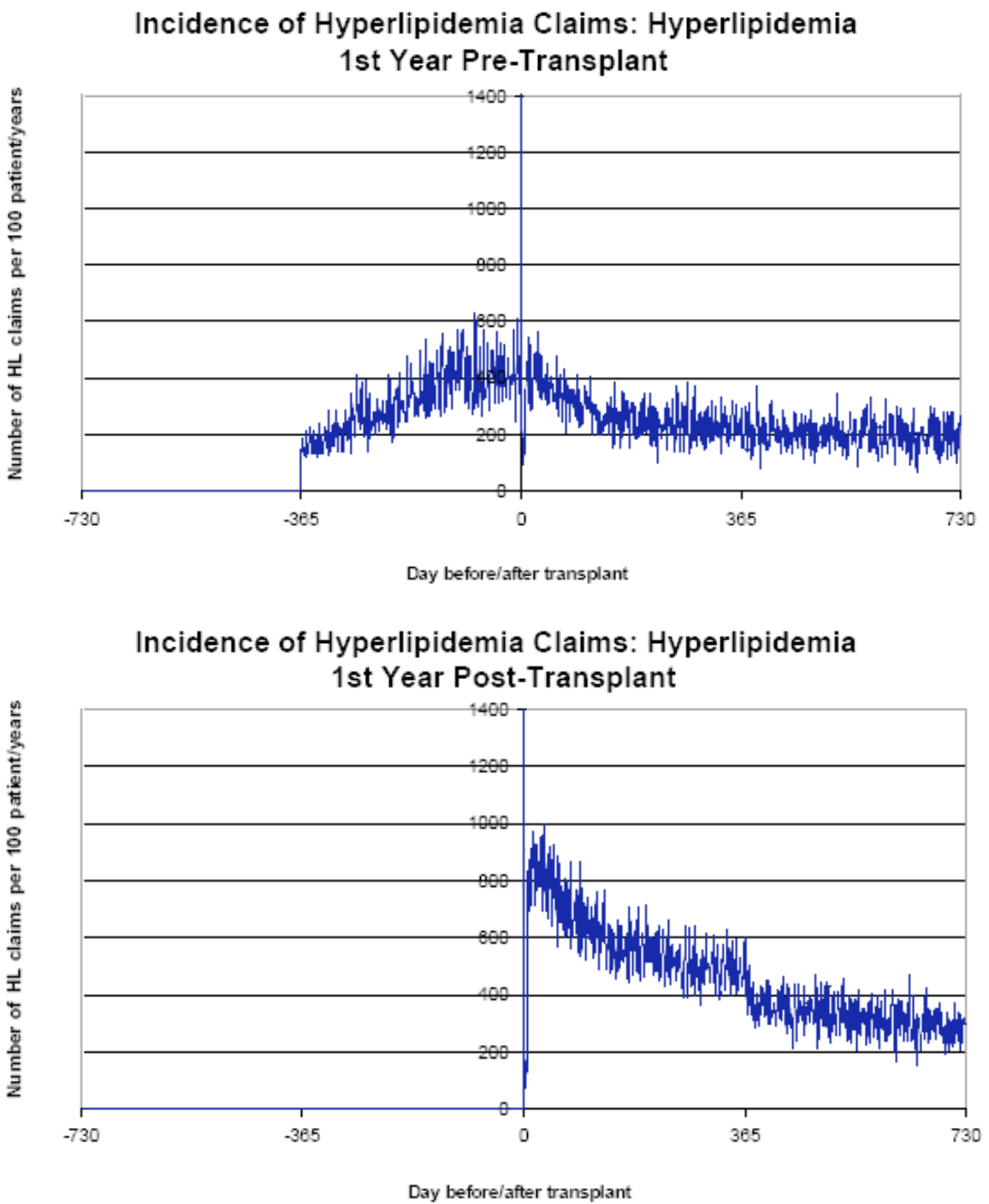

Fig. (1). Hyperlipidemia claims per 100 patient years among patients with NOHL first year pre-transplant and first year post-transplant. 
the date on which the second ICD-9-CM code between 272.0 and 272.4 was reported on any claim after one year's experience without any such claim. These ICD-9-CM codes correspond to "Pure Hypercholesterolemia" and "Mixed Hyperlipidemia", respectively. The analysis is restricted to Medicare patients, and therefore non-Medicare claims are not captured in the current study.

We constructed five cohorts according to key time points and the occurrence of NOHL in the identified subjects. The key time points were the day of transplant ("Day 0"), 2 years (730 days) before transplant, 1 year (365 days) before transplant, 1 year (365 days) after transplant, and 2 years (730 days) after transplant. Four time periods were defined and used to divide the groups of subjects with NOHL further into the following four cohorts: 1. NOHL within the second year before transplant; 2 . NOHL within the first year before transplant; 3. NOHL within the first year after transplant; and 4 . NOHL within the second year after transplant, with $5,222,4,346,7,438$, and 1,860 patients followed at transplantation, respectively. The fifth cohort included those transplant subjects who had no hyperlipidemia diagnosis during any of the four years, with 19,040 patients followed after transplantation.

Ideally this fifth cohort would be comprised of patients with no hyperlipidemia onset at all. However, given our cohort definitions it is possible that these patients may have had hyperlipidemia diagnosed prior to the two year pretransplant period studied. There is no variable provided in the USRDS data that defines the patient's hyperlipidemia status at transplant, and some patients are not followed prior to two years pre-transplant. Therefore, the best definition we could provide was the absence of hyperlipidemia in the physician/supplier and institutional claims.

The incidence of hyperlipidemia claims was plotted for each day pre- and post-transplant. Average accumulated post-transplant costs were calculated using a method similar to that of Kaplan-Meier survival curves. Specifically, the Average Accumulated Medicare Payments (AAMPs) for the first day post-transplant were set to $\$ 0$ since no patient was discharged that quickly. For each subsequent day " $\mathrm{t}$ ", the average accumulated payments equaled the average accumulated payments of the previous day plus the average incremental payments on day " $t$ ". The average incremental payments on day " $t$ " were calculated as the total Medicare payments made on day " $\mathrm{t}$ " $\left(\mathrm{MP}_{\mathrm{t}}\right)$ divided by the number of individuals remaining uncensored on day " $\mathrm{t}$ " $\left(\mathrm{n}_{\mathrm{t}}\right)$ :

$$
\operatorname{AAMP}_{t}=\operatorname{AAMP}_{t-1}+\left(M_{t} / n_{t}\right)-730>t>730
$$

We also calculated the graft survival rates over the two years following transplantation using Kaplan-Meier graft survival curves.

\section{RESULTS}

\section{Cohort Characteristics and Incidence}

The five cohorts used in this study differed with respect to various recipient, donor, and procedure characteristics on univariate analyses (Table 1). Patients with NOHL during the second pre-transplant year peaked at 554 diagnoses per 100 patient years and returned to a baseline of 224 by the end of the second post-transplant year. Patients with NOHL identified in the first year before transplantation peaked at 617 hyperlipidemia diagnoses per 100 person years shortly after transplantation and returned to a baseline of approximately 209 diagnoses per 100 person years in the second post-transplant year (Fig. 1). Patients with NOHL during the first post-transplant year peaked at 994 and had not returned to the 209 baseline by the end of the second post-transplant year (Fig. 1). Patients with NOHL during the second posttransplant year peaked at 714 diagnoses per 100 person-years by the end of the second post-transplant year. These results show that patients with hyperlipidemia identified before transplantation had fewer hyperlipidemia diagnoses after transplantation than did those with NOHL post-transplantation. These incidence rates suggested by the claims data are somewhat lower than those observed by Tse et al. in single center studies [13]. While NOHL is less prevalent among wait-listed patients, we found a non-zero hyperlipidemia incidence rate pre-transplant, consistent with Tse et al. [13].

\section{Hyperlipidemia and Average Accumulated Medicare Payments (AAMPs)}

The AAMPs represent the accumulation of all Medicare payments for transplant recipients in each cohort (Fig. 2). Compared to patients without NOHL during the two years before or two years after transplantation, patients with NOHL in the second year before transplantation cost Medicare an additional $\$ 15,228(\mathrm{p}<0.001)$ in the two years before transplantation and an additional $\$ 14,673(\mathrm{p}<0.001)$ by the end of the second year post-transplant. For patients who developed NOHL during the first year before transplantation, the additional Medicare payments were $\$ 2,443$ in the two years before transplantation and $\$ 6,935$ in the two years following transplantation $(\mathrm{p}<0.001)$. Patients who developed NOHL during the first post-transplant year cost Medicare an additional $\$ 2,722(p<0.001)$ by the end of the second posttransplant year. There was no significant cost difference between patients without NOHL and patients with NOHL during the second post-transplant year. These results suggest that early onset hyperlipidemia is particularly expensive. The AAMP cumulative cost curves used to measure the costs associated with the timing of hyperlipidemia onset do not allow for the identification of specific factors that are responsible for the increased costs.

\section{Confirmatory Multivariate Regression}

Multivariate regression analyses controlling for significant donor, recipient, and procedure characteristics as described in Table $\mathbf{1}$ confirmed the cost differences (Table 2). Stepwise regression results showed that developing hyperlipidemia during the second year pre-transplant cost Medicare an additional $\$ 8,961(\mathrm{p}<0.001)$ by the end of the second post-transplant year compared to no NOHL. Similarly, NOHL during the first pre-transplant year cost an additional $\$ 4,129(p<0.001)$. These estimates were smaller in magnitude than those implied by Fig. (2), as might be expected given that patients who developed hyperlipidemia were more likely to have other costly diseases, such as diabetes, cardiovascular disease, or peripheral artery disease. The two-year accumulated costs of patients with NOHL during the first or second post-transplant years were not significantly different 
Table 1. Descriptive Statistics of Hyperlipidemia Cohorts

\begin{tabular}{|c|c|c|c|c|c|c|}
\hline Variable & $\begin{array}{c}\text { NOHL } 2^{\text {nd }} \text { Year before } \\
T x\end{array}$ & $\begin{array}{l}\text { NOHL } \\
1^{\text {st }} \text { year } \\
\text { before } \\
\text { Tx }\end{array}$ & $\begin{array}{l}\text { NOHL } \\
1^{\text {st }} \text { Year } \\
\text { after Tx }\end{array}$ & $\begin{array}{c}\text { NOHL } \\
2^{\text {nd }} \text { Year after } \\
\text { Tx }\end{array}$ & $\begin{array}{l}\text { No } \\
\text { NOHL }\end{array}$ & $\begin{array}{c}\text { Significance of } \\
\text { Overall Differences }\end{array}$ \\
\hline Recipient age $<=17$ & 0.38 & 1.10 & 0.81 & 1.56 & 6.29 & $* *$ \\
\hline $45<=$ Recipient age $<60$ & 34.76 & 35.43 & 37.12 & 36.99 & 30.13 & $* *$ \\
\hline Recipient age $>=60$ & 32.98 & 28.92 & 21.75 & 18.28 & 15.81 & $* *$ \\
\hline Recipient male & 58.89 & 58.15 & 57.53 & 58.39 & 61.47 & $* *$ \\
\hline Recipient black & 28.46 & 24.90 & 27.80 & 28.98 & 31.87 & $* *$ \\
\hline Recipient Hispanic & 15.35 & 13.30 & 13.84 & 13.94 & 14.20 & \\
\hline Living donor & 18.17 & 30.72 & 25.93 & 26.56 & 27.02 & $* *$ \\
\hline Donor age (years) & 38.08 & 38.21 & 37.72 & 37.65 & 36.66 & $* *$ \\
\hline Donor male & 55.61 & 52.36 & 54.51 & 54.45 & 54.07 & $* *$ \\
\hline Donor black & 11.82 & 11.62 & 11.94 & 13.82 & 14.18 & $* *$ \\
\hline Donor Hispanic & 13.39 & 11.34 & 12.19 & 12.99 & 12.30 & $*$ \\
\hline Cyclosporine & 49.39 & 53.34 & 55.65 & 58.76 & 46.04 & $* *$ \\
\hline Azathioprine & 10.82 & 14.36 & 13.51 & 21.18 & 24.22 & $* *$ \\
\hline Mycophenolate mofetil & 70.93 & 68.87 & 66.43 & 62.37 & 59.16 & $* *$ \\
\hline Warm ischemia time (minutes) & 24.50 & 22.49 & 23.12 & 22.67 & 23.39 & \\
\hline Cold ischemia time (hours) & 19.56 & 18.99 & 19.01 & 18.90 & 18.87 & \\
\hline Number of HLA mismatches & 3.39 & 3.11 & 3.26 & 3.23 & 3.19 & $* *$ \\
\hline Hepatitis $\mathrm{C}$ at $\mathrm{tx}$ & 5.78 & 5.04 & 4.97 & 5.91 & 6.20 & $* *$ \\
\hline Diabetes as a cause of ESRD & 28.88 & 28.92 & 21.61 & 21.67 & 18.36 & $* *$ \\
\hline History of diabetes & 33.86 & 33.85 & 25.21 & 23.49 & 19.42 & $* *$ \\
\hline History of cardiovasc. disease & 3.12 & 2.81 & 2.23 & 1.72 & 1.75 & $* *$ \\
\hline History of peripheral vasc. disease & 5.74 & 4.79 & 3.20 & 3.98 & 3.04 & $* *$ \\
\hline Donor CMV+ / Recipient CMV + & 46.45 & 43.08 & 41.27 & 42.10 & 41.10 & $* *$ \\
\hline Donor CMV- / Recipient CMV + & 16.49 & 17.62 & 18.65 & 19.59 & 19.68 & * \\
\hline Donor CMV+ / Recipient CMV - & 24.6 & 25.82 & 24.66 & 23.05 & 23.29 & $* *$ \\
\hline \multirow[t]{2}{*}{$\begin{array}{l}\text { Panel reactive antibody percent } \\
\qquad>=50\end{array}$} & 4.08 & 3.29 & 3.83 & 3.60 & 4.42 & $* *$ \\
\hline & $\mathrm{n}=5,222$ & $\mathrm{n}=4,346$ & $\mathrm{~N}=7,438$ & $\mathrm{n}=1,860$ & $\mathrm{n}=19,040$ & \\
\hline
\end{tabular}

When constructing explanatory variables, missing values were coded as the absence of the status or condition. $*$ and $* *$ denote statistical significance at the $95 \%$ and $99 \%$ confidence level, respectively. Table values are percentages unless otherwise labeled.

from the costs of patients without NOHL $(\mathrm{P}=0.373,0.571$ respectively).

\section{Association Between NOHL on Graft Survival}

Kaplan-Meier plots depicting the graft survival rates for each of the five cohorts, are shown in Fig. (3). Patients who did not develop NOHL during the study period had lower graft survival rates at 2 years post-transplant than patients who did develop NOHL during the four-year period. Thus, among those who developed NOHL during the study period, patients with NOHL in the second year pre-transplant experienced a two year graft survival rate of $80.25 \%$ compared to $86.60 \%$ among those with NOHL in the second year post-transplant. The graft survival rate among patients with NOHL in the first pre-transplant year was $83.66 \%$, while the graft survival rate among patients with NOHL in the first post-transplant year was $85.94 \%$. Importantly, the results from "death censored" models were not significantly different.

Cox proportional hazard models confirmed the KaplanMeier plots. Hazard ratios indicated that NOHL is associated with a lower occurrence of graft failure. Recipients who were older, black, had histories of other diseases (such as diabetes, hepatitis $\mathrm{C}$, peripheral vascular disease, or cardiovascular disease), were recipients of a black donor, were highly HLA-sensitized recipients with panel reactive antibodies greater than $50 \%$, had received cadaveric donors, 


\section{Hyperlipidemia: Average Accumulated Medicare Payments}

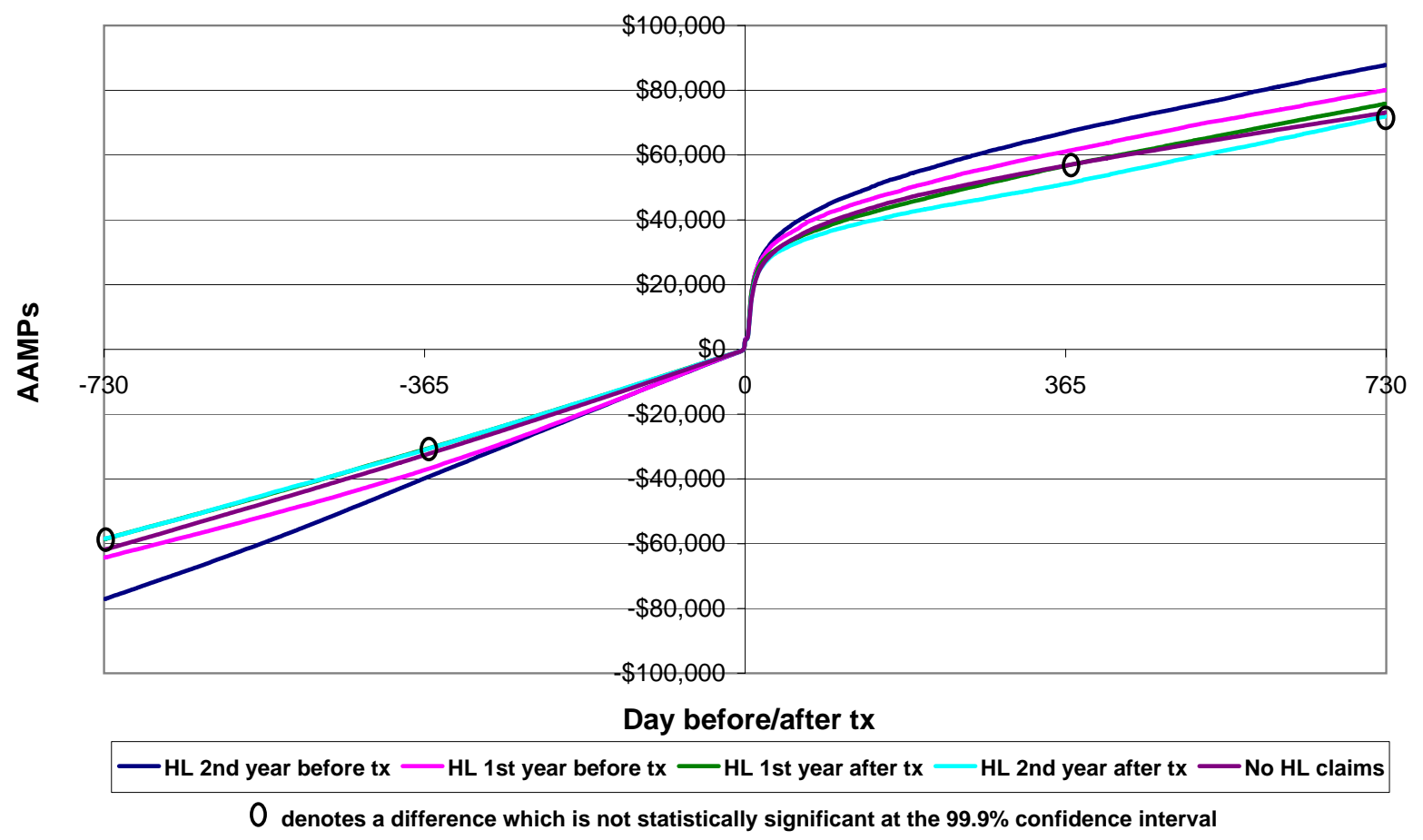

Fig. (2). Average accumulated Medicare payments.

Note: The green line denoting "HL 1 st year after tx" is almost directly underneath the light blue line denoting "HL 2nd year after tx".

\section{Graft Survival by Hyperlipidemia Onset Time}

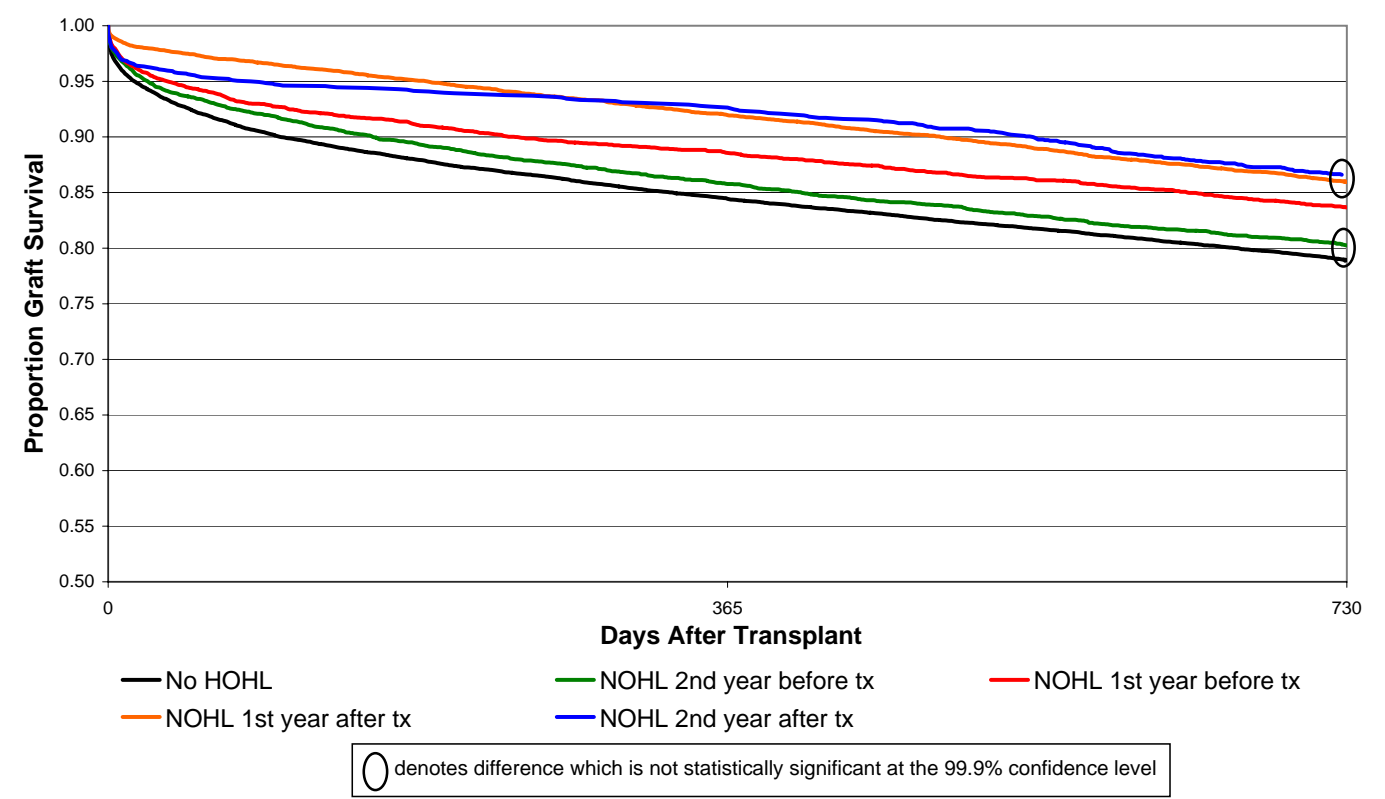

Fig. (3). Graft survival by hyperlipidemia onset time.

or were donor and recipient seropositive for cytomegalovirus had higher probabilities of graft failure. Recipients who received azathioprine, cyclosporine or mycophenolate com- pared to those who did not receive each as well as those who received transplants more recently had higher rates of graftsurvival. 
Table 2. Confirmatory Stepwise Multivariate Regression: Significant Determinants of Two-Year Accumulated Medicare Payments

\begin{tabular}{|c|c|c|}
\hline Variable & $\begin{array}{c}\text { Parameter } \\
\text { Estimate in \$ }\end{array}$ & P-Value \\
\hline Intercept & 47,824 & $<.0001$ \\
\hline NOHL 2nd year pre-transplant & 8,961 & $<.0001$ \\
\hline NOHL 1st year pre-transplant & 4,129 & 0.0007 \\
\hline Recipient age $\leq 17$ & 9,708 & $<.0001$ \\
\hline Recipient age between 45 and 60 & 2,439 & 0.0041 \\
\hline Recipient age $\geq 60$ & 5,280 & $<.0001$ \\
\hline Donor age & 286 & $<.0001$ \\
\hline Recipient black & 5,791 & $<.0001$ \\
\hline Hepatitis $\mathrm{C}$ at transplant & 12,103 & $<.0001$ \\
\hline Donor black & 3,969 & 0.0005 \\
\hline History of diabetes & 5,646 & 0.0001 \\
\hline $\begin{array}{l}\text { History of peripheral vascular } \\
\text { disease }\end{array}$ & 6,300 & 0.0011 \\
\hline $\begin{array}{c}\text { History of cardiovascular } \\
\text { disease }\end{array}$ & 7,203 & 0.0052 \\
\hline $\begin{array}{c}\text { Panel reactive antibody percent } \\
\qquad \leq 50\end{array}$ & 9,504 & $<.0001$ \\
\hline Diabetes as cause of ESRD & 4,183 & 0.0042 \\
\hline Number of HLA mismatches & 1,049 & $<.0001$ \\
\hline $\begin{array}{c}\text { Azathioprine (compared to "no } \\
\text { Aza") }\end{array}$ & $-5,079$ & $<.0001$ \\
\hline $\begin{array}{c}\text { Cyclosporine (Compared to "no } \\
\text { CsA") }\end{array}$ & $-5,417$ & $<.0001$ \\
\hline Cadaveric donor & 5,963 & $<.0001$ \\
\hline Donor CMV+/Recipient CMV- & 2,154 & 0.0215 \\
\hline Recipient male & $-2,377$ & 0.0016 \\
\hline $\begin{array}{c}\text { Year } 1996 \text { (compared to year } \\
1995)\end{array}$ & 3,963 & $<.0001$ \\
\hline $\begin{array}{c}\text { Year } 2000 \text { (compared to year } \\
1995)\end{array}$ & $-2,805$ & 0.0375 \\
\hline
\end{tabular}

Variables found to be insignificant were "NOHL 1st year post transplant", "NOHL 2nd year post transplant", recipient Hispanic, donor male, donor Hispanic, Mycofenolate mofetil (compared to "no MMF), warm ischemia time, and cold ischemia time . (Adjusted $\mathrm{R}^{2}=0.0401$ ).

Our result that NOHL was associated with lower occurrences of graft failure is consistent with prior research that suggested that this association may be attributed to inflammation and malnutrition, which are associated with lower lipid levels and adverse outcomes [14,15]. Among those patients with NOHL during our study period, earlier onset of NOHL was associated with higher rates of graft failure. Alternatively, if patients without NOHL are being treated for more serious co-morbidities consistent with higher rates of graft failure, such as hepatitis or pneumonia, and clinicians are not billing for the hyperlipidemia treatment, then our use of claims data will miss these potential cases of NOHL.

\section{CONCLUSIONS}

This study compared costs and graft-survival related to NOHL based on the time of onset relative to transplantation.
Our results indicated that patients diagnosed with NOHL in the second pre-transplant year incurred additional two-year accumulated Medicare payments of $\$ 14,673$ compared to patients with no hyperlipidemia diagnoses. Patients diagnosed with NOHL in the first pre-transplant year incurred additional two-year accumulated Medicare payments of $\$ 6,935$. Although NOHL was associated with increased preand post-transplant costs, patients diagnosed with NOHL between the second year before and second year after transplantation experienced higher graft survival rates than those with no NOHL diagnoses during that time period.

Our findings are important. Development of hyperlipidemia is expensive. At least one new immunosuppressive agent, sirolimus, is associated with increased incidences of hyperlipidemia, but improvements in short-term renal function and no significant impact on cardiovascular mortality $[16,17]$. Therefore, in assessing the costs and benefits of these immunosuppressive agents, researchers must take into account the costs of the associated increases in the incidence of hyperlipidemia. The impact on long-term costs and outcomes remains to be determined.

Our study has limitations. It was retrospective, relied on registry data, and did not assess costs and outcomes beyond 2 -years post-transplantation. Claims data may not reflect the true incidence of hyperlipidemia, especially if patients are being screened and treated for hyperlipidemia in conjunction with other treatments. The hyperlipidemia incidence found in our study was lower than those typically found in single center studies, however it was not zero. The diagnostic codes for our study did not take into consideration the impact of dyslipidemia such as low high-density lipoproteinemia, which is a major factor for cardiovascular disease. The prevalence of diagnosed dyslipidemia was relatively low compared to hyperlipidemia. We did not compare the impact of maintenance peritoneal dialysis and hemodialysis on costs or graft-survival. However, we doubt that this would have been significant overall given the relatively small percentage of patients receiving peritoneal dialysis in the U.S. We also did not directly compare the use of cyclosporine to tacrolimus for maintenance immunosuppression. Use of cyclosporine is associated with more hyperlipidemia than use of tacrolimus. However we did compare the use of cyclosporine to those who did not receive cyclosporine. Future work could consider differences in costs and outcomes by type of medication rather than by the timing of hyperlipidemia onset. Finally, the data do not allow assessment of the use of "statins" which may impact costs and outcomes.

Although NOHL was associated with increased pre- and post-transplant costs, patients diagnosed with NOHL between the second year before and second year after transplantation experienced higher graft-survival rates than those with no-NOHL during that time period. Prior studies have attributed this association to malnutrition and inflammation, which may lower cholesterol levels but have adverse effects on graft survival.

\section{ACKNOWLEDGEMENT}

This research was conducted under an unrestricted grant provided by Pfizer, Inc. 


\section{ABBREVIATIONS}

AAMP $=$ Average Accumulated Medicare Payments

ESRD $=$ End Stage Renal Disease
NOHL $=$ New Onset Hyperlipidemia

PTHL $=$ Post Transplant Hyperlipidemia

USRDS $=$ United States Renal Data System

\section{APPENDIX}

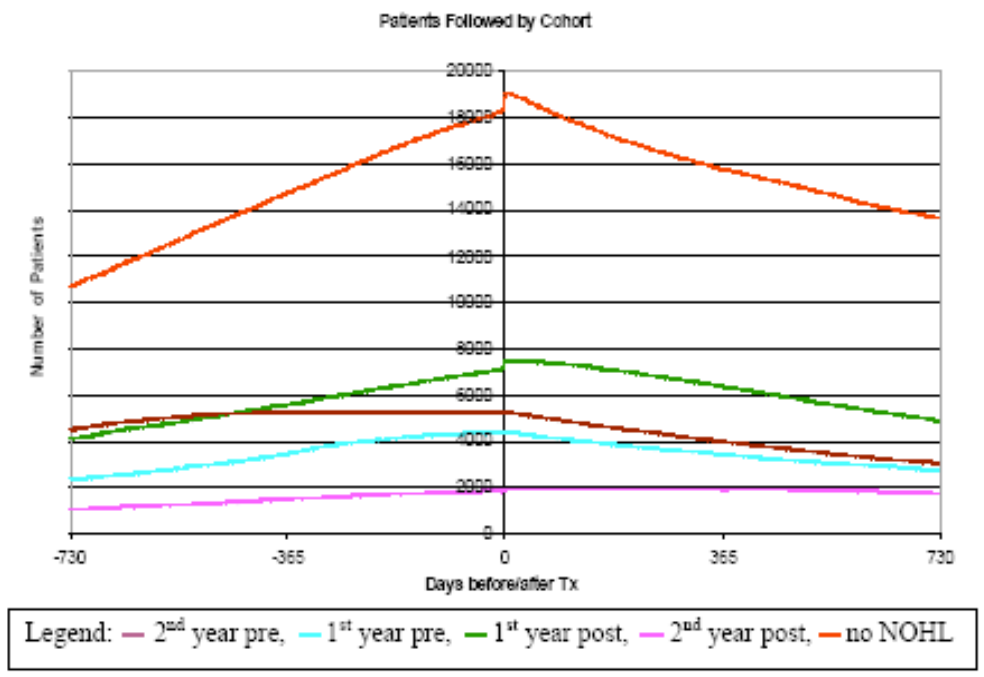

Fig. (A1). Number of patients followed by the timing of NOHL.

Incidence of Hyperlipidemia Claims: Hyperlipidemia 2nd Year Pre-Transplant

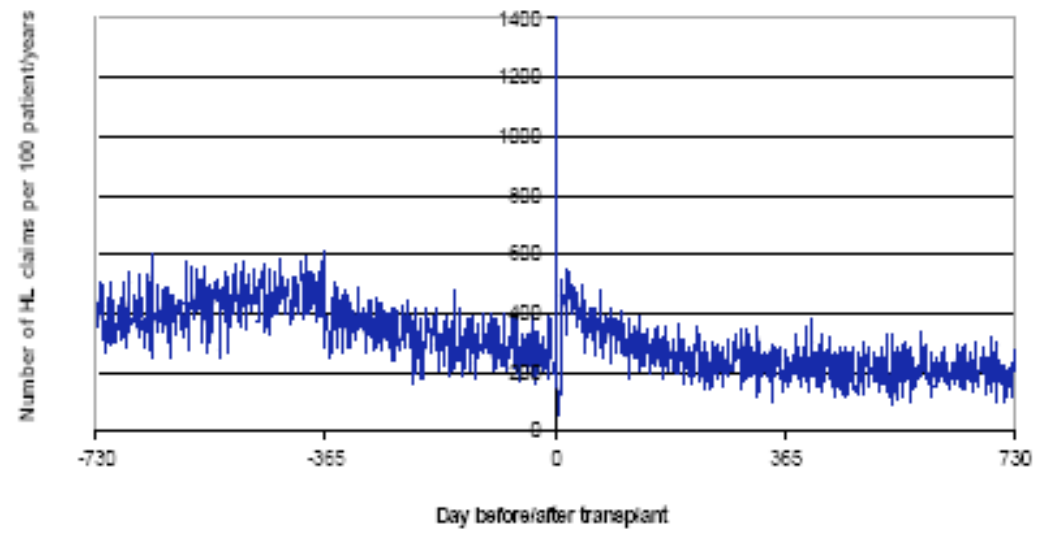

Fig. (A2). Hyperlipidemia claims per 100 patient years among patients with NOHL second year pre-transplant.

\section{Incidence of Hyperlipidemia Claims: Hyperlipidemia} 2nd Year Post-Transplant

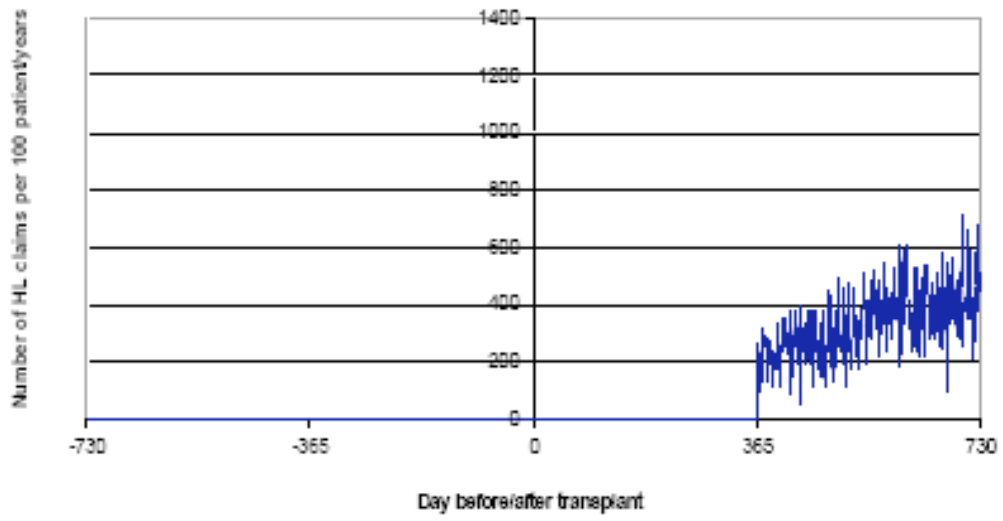

Fig. (A3). Hyperlipidemia claims per 100 patient years among patients with NOHL second year post-transplant. 
Table A1. Average Accumulated Medicare Payments

\begin{tabular}{|c|c|c|c|c|}
\hline & Day $-\mathbf{7 3 0}$ & Day $\mathbf{- 3 6 5}$ & Day 365 & Day 730 \\
\hline \multirow{2}{*}{ NOHL 2nd year before tx } & $-\$ 77,065$ & $-\$ 39,814$ & $\$ 67,487$ & $\$ 87,783$ \\
\hline & $(4,463)$ & $(5,222)$ & $(3,947)$ & $(3,008)$ \\
\hline \multirow{2}{*}{ NOHL 1 st year before tx } & $-\$ 64,280$ & $-\$ 37,249$ & $\$ 61,508$ & $\$ 80,045$ \\
\hline & $(2,315)$ & $(3,427)$ & $(3,398)$ & $(2,717)$ \\
\hline NOHL 1 st year after tx & $-\$ 58,574$ & $-\$ 30,912$ & $\$ 56,670$ & $\$ 75,832$ \\
\hline \multirow{2}{*}{ NOHL 2nd year after tx } & $-\$ 58,457$ & $-\$ 31,046$ & $\$ 51,110$ & $\$ 71,943$ \\
\hline & $(1,028)$ & $(1,443)$ & $(1,860)$ & $(1,710)$ \\
\hline \multirow{2}{*}{ No NONo NOHL } & $-\$ 61,837$ & $-\$ 32,636$ & $\$ 56,751$ & $\$ 73,110$ \\
\hline & $(10,665)$ & $(14,699)$ & $(15,773)$ & $(13,642)$ \\
\hline
\end{tabular}

The number of patients followed in each cohort on each day is given in parentheses.

Table (A2). Cox Proportional Hazard Estimates of Graft Survival

\begin{tabular}{|c|c|c|c|}
\hline Variable & Parameter Estimate & p-Value & $\begin{array}{c}\text { Hazard Ratio } \\
\text { 95\% Confidence Interval }\end{array}$ \\
\hline NOHL 2nd year pre-transplant & -0.16855 & 0.0003 & 0.7710 .925 \\
\hline NOHL 1st year pre-transplant & -0.28191 & $<.0001$ & 0.6800 .837 \\
\hline NOHL 1st year post-transplant & -0.55419 & $<.0001$ & 0.5250 .629 \\
\hline NOHL $2^{\text {nd }}$ year post-transplant & -0.59274 & $<.0001$ & 0.4740 .644 \\
\hline Recipient age between 45 and 60 & 0.15377 & $<.0001$ & 1.0881 .250 \\
\hline Recipient age $\geq 60$ & 0.39089 & $<.0001$ & 1.3661 .600 \\
\hline Donor age & 0.01127 & $<.0001$ & 1.0091 .013 \\
\hline Recipient black & 0.19508 & $<.0001$ & 1.1381 .298 \\
\hline Hepatitis $\mathrm{C}$ at transplant & 0.18395 & 0.0009 & 1.0781 .340 \\
\hline Donor black & 0.18728 & $<.0001$ & 1.1071 .314 \\
\hline History of diabetes & 0.1325 & 0.0002 & 1.0651 .224 \\
\hline History of peripheral vascular disease & 0.20279 & 0.0046 & 1.0641 .409 \\
\hline History of cardiovascular disease & 0.2667 & 0.0029 & 1.0961 .556 \\
\hline Panel reactive antibody percent $\geq 50$ & 0.42117 & $<.0001$ & 1.3421 .730 \\
\hline Number of HLA mismatches & 0.07301 & $<.0001$ & 1.0571 .095 \\
\hline Azathioprine (compared to "no Aza") & -0.26637 & $<.0001$ & 0.7040 .834 \\
\hline Cyclosporine (compared to "no CsA") & -0.14858 & $<.0001$ & 0.8110 .916 \\
\hline Mycophenolate mofetil (compared to "no MMF") & -0.29634 & $<.0001$ & 0.6910 .800 \\
\hline Cadaveric donor & 0.43375 & $<.0001$ & 1.4031 .697 \\
\hline Donor CMV+/Recipient CMV- & 0.10343 & 0.0061 & 1.0301 .194 \\
\hline Donor male & -0.14609 & $<.0001$ & 0.8140 .917 \\
\hline Year 2000 (compared to year 1995) & -0.22327 & 0.0003 & 0.7090 .902 \\
\hline Year 2001 (compared to year 1995) & -0.23431 & 0.0011 & 0.6870 .910 \\
\hline
\end{tabular}

$\mathrm{N}=25,055$. Variables listed in Table 1 that do not appear in this model were found to be insignificant. (Overall model significance $\mathrm{p}<0.0001$ ).

\section{REFERENCES}

[1] Wanner C, Krane V, Metzger T, Quaschning T. Lipid changes and statins in chronic renal insufficiency and dialysis. J Nephrol 2001; 14(Suppl 4): S76-80.

[2] Aalamian Z. Reducing adverse effects of immunosuppressive agents in kidney transplant recipients. Prog Transplant 2001; 11: 271-82.

[3] Shoji T, Ishimura E, Inaba M, Tabata T, Nishizawa Y. Atherogenic lipoproteins in end-stage renal disease. Am J Kidney Dis 2001; 38 (Suppl 1): S30-3.
[4] Wissing KM, Abramowicz D, Broeders N, Vereerstraeten P. Hypercholesterolemia is associated with increased kidney graft loss caused by chronic rejection in male patients with previous acute rejection. Transplantation 2000; 70: 464-72.

[5] Wheeler DC, Steiger J. Evolution and etiology of cardiovascular diseases in renal transplant recipients. Transplantation 2000; 70 (Suppl): SS41-45.

[6] Woodward RS, Schnitzler MA, Baty J, et al. Incidence and cost if new onset diabetes mellitus among U.S. wait-listed and transplanted renal allograft recipients. AM J Transplant 2003; 3(5): 590-8. 
[7] Kutinova A, Woodward RS, Ricci JF, Brennan DC. The incidence and costs of sepsis and pneumonia before and after renal transplantation in the United States. AM J Transplant 2006; 6(1): 129-39.

[8] Satterthwaite R, Aswad S, Sunga V, et al. Incidence of new-onset hypercholesterolemia in renal transplant patients treated with FK506 or cyclosporine. Transplantation 1998; 65: 446-9.

[9] Jensik SC. Tacrolimus (FK506) in kidney transplantation: threeyear survival results of the US multicenter, randomized, comparative trial. FK506 Kidney Transplant Study Group. Transplant Proc 1998; 30: 1216-8.

[10] Mayer AD. Four-year follow-up of the european tacrolimus multicenter renal study. Transplant Proc 1999; 31: 27S-8S.

[11] Chan CM. Hyperlipidemia in chronic kidney disease. Ann Acad Med Singapore 2005; 34(1): 31-5.

[12] United States Renal Data System (USRDS). USRDS Data CDROMS. AUSRDS, Minneapolis, MN: May 2000.
[13] Tse KC, Lam MF, Yip PS, Li FK, Lai KN, Chan TM. A long-term study on hyperlipidemia in stable renal transplant recipients. Clin Transplant 2004; 18(3): 274-80.

[14] Yongmei L, Coresh J, Eustace JA, et al. Association between cholesterol Level and Mortalit in Dialysis Patients. JAMA 2004; 291: 451-59.

[15] Weiner DE, Sarnak MJ. Managind dyslipidemia in Chronic Kidney Disease. J Gen Intern Med 2004; 19: 1045-52.

[16] Hocker B, Feneberg R, Kopf S, et al. SRL-based immunosuppression vs. CNI minimization in pediatric renal transplant recipients with chronic CNI nephrotoxicity. Pediatr Transplant 2006; 10(5): 593-601.

[17] Boratynska M, Banasik M, Watorek E, et al. Conversion to sirolimus from cyclosporine may induce nephritic proteinuria and progressive deterioration of renal function in chronic allograft nephropathy patients. Transplant Proc 2006; 38(1): 101-4.

(C) Woodward et al.; Licensee Bentham Open.

This is an open access article licensed under the terms of the Creative Commons Attribution Non-Commercial License (http://creativecommons.org/ licenses/by-nc/3.0/), which permits unrestricted, non-commercial use, distribution and reproduction in any medium, provided the work is properly cited. 